\title{
Message from the Editors
}

\author{
ADAM WIERMAN, California Institute of Technology \\ ADITYA AKELLA, University of Wisconsin-Madison
}

This issue marks the completion of the first year of the Proceedings of the ACM on Measurement and Analysis of Computing Systems (POMACS). POMACS was among the first three journals joining the recently launched Proceedings of the ACM (PACM) series, and with this issue POMACS has now published over 80 papers.

The goal of the PACM series is to showcase the highest quality research conducted in diverse areas of computer science, as represented by the ACM Special Interest Groups (SIGs). ACM POMACS focuses on the computer systems measurement and performance evaluation community and operates in close collaboration with the Special Interest Group SIGMETRICS. In fact, all the papers in the last three issues of POMACS will be presented during the SIGMETRICS annual conference this summer.

The past four decades have seen enormous changes in the field of computer science, but the importance of measurement, modeling, and performance evaluation remains as critical as ever, and the papers that have appeared in POMACS during the past year reflect the ability of the journal to attract high-quality submissions while evolving with the broad interests of the performance evaluation community. The last three issues have included papers on topics that have been mainstays in the performance evaluation community, including load balancing, scheduling, resource allocation, and performance measurement; as well as emerging new application areas that have become prominent in recent years, such as the sharing economy, cryptocurrencies, network science, and machine learning.

POMACS publishes three issues yearly. Each issue contains papers selected by the editorial board via a review process that follows a hybrid of the conference and journal models, with rigorous reviews conducted by the editorial board whereby each paper is either conditionally accepted (with a minor revision and shepherded), allowed a "one-shot" revision (major or minor, with a limited time window for revision and re-submission), or rejected (with re-submission to the journal allowed after a year or more from the time of submission). Over the three issues during which we have served as editors, POMACS has published 54 papers out of 270 submissions (58 to the Summer deadline, 92 to the fall deadline, and 120 to the winter deadline). Each paper received 3-7 reviews in two rounds. Unlike a typical journal, all reviews were completed by the 65 members of our editorial board, except for a handful of papers for which we sought an external expert opinion.

Many people contributed to the success of POMACS during the last year. First, we would like to thank all the authors for contributing their work. We would also like to thank the editorial board members for staying responsive and positive throughout the year. We would additionally like to thank last year's editors (Augustin Chaintreau, Leana Golubchik, and Zhi-Li Zhang) for being constantly available and willing to share their experiences.

We hope that you enjoy this issue of POMACS and that you consider contributing your work to future issues!

Authors' addresses: Adam Wierman, California Institute of Technology, adamw@caltech.edu; Aditya Akella, University of Wisconsin-Madison, akella@cs.wisc.edu. 